\title{
Evaluation of serum interleukin-10 levels in hepatocellular carcinoma patients: a systematic review and meta-analysis
}

\author{
Ebrahim Shakiba', Mazaher Ramezani², Masoud Sadeghi \\ 'Department of Clinical Biochemistry, Kermanshah University of Medical Sciences, Kermanshah, Iran \\ 2Molecular Pathology Research Center, Emam Reza University Hospital, Kermanshah University of Medical Sciences, Kermanshah, Iran \\ ${ }^{3}$ Medical Biology Research Center, Kermanshah University of Medical Sciences, Kermanshah, Iran
}

\begin{abstract}
Aim of the study: This meta-analysis aimed to evaluate serum IL-10 levels in HCC patients.

Material and methods: The three databases PubMed, Scopus and Web of Science were checked on July 2017 for assessment of IL-10 levels in hepatocellular carcinoma (HCC) patients compared with healthy controls in publications with an English abstract. A random-effect meta-analysis was performed using mean difference (MD) and $95 \%$ confidence intervals (Cls). Also, publication bias was evaluated through funnel plot analysis with the Begg's and Egger's tests.

Results: Out of 171 studies searched in the databases, nine studies were included in the meta-analysis. The pooled analysis with continuous data concluded that IL-10 level in the patients was significantly higher than the controls ( $\mathrm{MD}=6.96 ; 95 \% \mathrm{Cl}=4.91-9.01 ; p<0.00001)$, in the $\mathrm{HCC}$ patients was significantly higher than the cirrhotic patients $(\mathrm{MD}=2.92 ; 95 \% \mathrm{Cl}=0.72-5.12 ; p=0.009)$, and was similar in the HCC patients compared with the patients with hepatitis (MD $=3.91 ; 95 \% \mathrm{Cl}=-4.25-12.07 ; p=0.35)$.

Conclusion: The increased IL-10 levels in the HCC patients compared with the cirrhotic patients and the healthy controls may show a significant role of this cytokine in the elevated risk of HCC, but the lack of significant difference in the levels between HCC and hepatitis makes it an unreliable tumor marker in the latter.
\end{abstract}

Key words: interleukin-10, hepatocellular carcinoma, cirrhosis, hepatitis.

\section{Address for correspondence}

Masoud Sadeghi, Medical Blology Research Center, Kermanshah University of Medical Sciences, 67145-1673 Kermanshah, Iran, phone: 00989185960644, e-mail: sadeghi_mbrc@yahoo.com

\section{Introduction}

Hepatocellular carcinoma (HCC) is one of the major malignant diseases worldwide, ranking as the fifth most common cancer [1] with poor prognosis and a low fiveyear survival rate [2] that often occurs in patients with chronic liver disease, due to hepatitis $\mathrm{B}$ virus (HBV) or hepatitis $\mathrm{C}$ virus (HCV) infection $[1,3]$. Cytokines play an important role in differentiation, maturation, and functional activation of immune cells [4]. Interleukin-10 (IL-10) is a pleiotropic cytokine produced by macrophages, T-helper 2 (Th2) cells and B-lymphocytes, and can stimulate and suppress the immune response. This interleukin has been shown to inhibit various immune reactions [5]. Serum IL-10 level has been reported to be significantly increased in patients with chronic hepatitis $\mathrm{C}$ and may be related to hepatocarcinogenesis with suppression of immune surveillance [6]. Therefore, it has been proposed that IL-10 plays a key role in the oncogenetic and metastatic ability of neoplasms [7]. An elevated level of IL-10 have been found in the plasma of patients with different histotypes of solid and hematopoietic tumors [8] and has an inhibitory effect on cancer immunosurveillance [9]. IL-10 has been assessed as a marker for HCC to predict postoperative recurrence of HCC $[7,9]$; however, it has 
not yet been validated for clinical use [7]. The aim of this meta-analysis was to evaluate serum IL-10 levels in HCC patients in comparison with healthy controls as well as hepatitis and cirrhotic patients.

\section{Material and methods}

\section{Search strategies}

We used the terms 'IL-10', 'interleukin-10' combined with 'liver cancer', 'HCC' and 'hepatocellular carcinoma 'in the three databases PubMed, Scopus and Web of Science in July 2017 for publications with an English abstract.

\section{Study selection}

One author (M.S.) searched the articles followed by the second author (M.R.) blinded to the first author. If there was any disagreement between the two authors, third author (E.S.) resolved the problem. All studies were searched for assessment of IL-10 levels in the HCC patients compared with the healthy controls, cirrhotic patients or patients with hepatitis. The inclusion criteria for the studies included: I) studies reporting

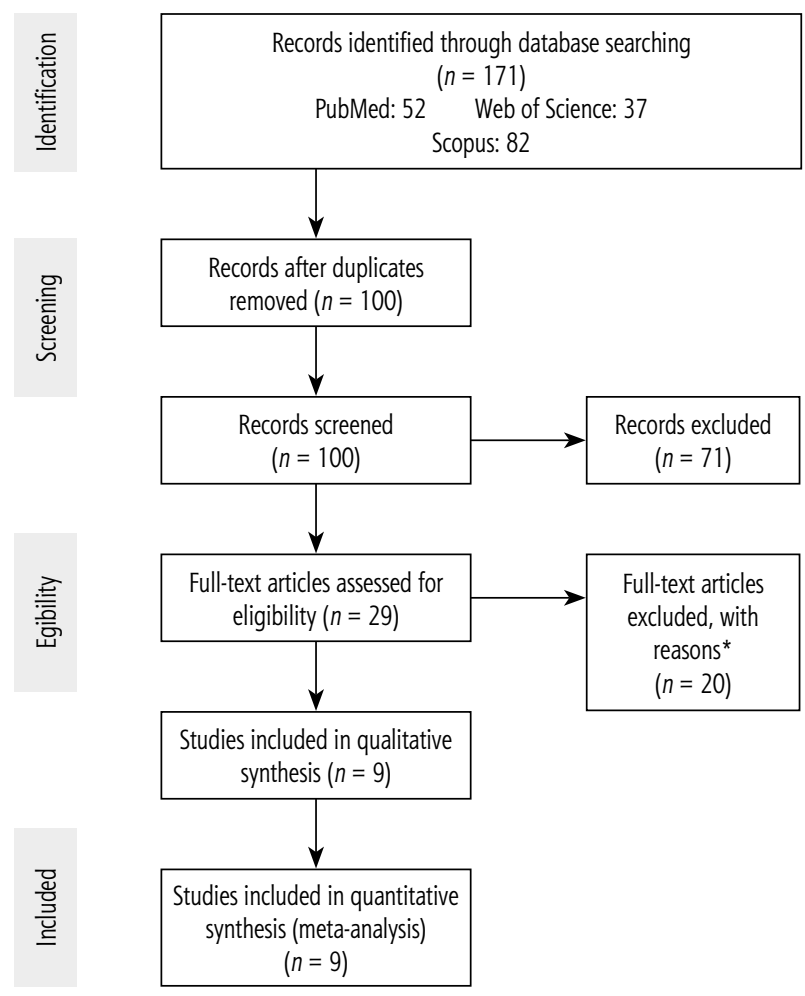

*1 study was review; 2 studies were conference paper; 3 had non-English full-text without needed information in English abstract; 12 studies didn't have control group; 2 studies report irrational mean of IL-10.

Fig. 1. Flowchart of the study the mean or median IL-10 level in HCC patients compared with the healthy controls, cirrhotic patients or patients with hepatitis; II) only studies with Englishlanguage abstract could be included; III) human studies; IV) HCC was confirmed histologically or based on biopsy or cytologic findings; V) a blood sample of IL-10 was taken after overnight fasting. The exclusion criteria were: I) the studies did not report the mean or median IL-10 level; II) data from incomplete reports (not sufficient information), review studies and conference papers were not eligible for this study.

\section{Data extraction}

The relevant data extracted from every study were the name of the author, year of publication, country, and number/age/male (\%) of the HCC, cirrhosis, hepatitis and healthy control groups. Level of IL-10 was determined by enzyme-linked immunosorbent assay.

\section{Statistical analysis}

A random-effect meta-analysis was performed by Review Manager 5.3 (RevMan 5.3, The Cochrane Collaboration, Oxford, United Kingdom) using mean difference (MD) and 95\% confidence intervals (CIs). Heterogeneity between the estimates was evaluated by the $\mathrm{Q}$ and $\mathrm{I}^{2}$ statistic where for the $\mathrm{Q}$ statistic, heterogeneity was considered if $p<0.1$ and $p$-value (2-sided) $<0.05$ was considered statistically significant in this meta-analysis. In addition, publication bias was evaluated through funnel plot analysis with the Begg's and Egger's tests. If the data were presented using the standard error (SE), then the formula

$$
S E=\frac{S D}{\sqrt{N}}
$$

was used to calculate standard deviation (SD). We used the formula for estimation of mean and SD if the study reported the median plus range [10]. The unit of measurement of IL-10 in this meta-analysis was $\mathrm{pg} / \mathrm{ml}$.

\section{Results}

Out of 171 studies searched in the databases after removing duplicate and non-relevant studies, 29 studies were assessed for eligibility, of which 20 studies were excluded based on exclusion criteria. Therefore, nine studies were included in the meta-analysis (Fig. 1).

The characteristics of the studies included in the meta-analysis are shown in Table 1 . Three studies were reported from Japan $[6,11,12]$, one from Korea [1], 
Table 1. The characteristics of the studies included in meta-analysis $(n=9)$

\begin{tabular}{|c|c|c|c|c|c|}
\hline \multirow[t]{2}{*}{ Study, year } & Country & HCC patients & Healthy controls & LC & $\mathrm{CH}$ \\
\hline & & ( $n /$ mean age, years/male \%) & ( $n /$ mean age, years/male $\%)$ & ( $n /$ mean age, years $/$ male $\%)$ & ( $n /$ mean age, years/male $\%)$ \\
\hline Kakumo, 1997 & Japan & $11 / 64 / 81.8$ & $11 / 45 / 63.6$ & $22 / 61 / 72.7$ & 28/54/71.4 \\
\hline Chau, 2000 & Taiwan & $67 / 63.4 / 89.6$ & $27 /-/-$ & - & - \\
\hline Hattori, 2003 & Japan & 74/median: 65/73 & 44/matched/- & 32/matched/- & - \\
\hline Ayada, 2006 & Japan & $8 / 56 / 75$ & $19 / 34 / 68.4$ & $12 / 53 / 83.3$ & $34 / 37 / 55.9$ \\
\hline Jang, 2012 & Korea & $110 / 58.6 / 71.8$ & $24 /-/-$ & - & - \\
\hline Wang, 2012 & China & 38/median: 53/82 & 30/median: 50/77 & - & - \\
\hline Othman, 2013 & Egypt & $20 / 60.1 / 60$ & $20 / 51.5 / 50$ & 20/55.6/75 & $20 / 55.1 / 65$ \\
\hline El-Emshaty, 2015 & Egypt & $15 / 57.1 / 86.7$ & $10 / 35.6 / 70$ & $16 / 46.1 / 75$ & $10 / 35.8 / 90$ \\
\hline Eltaher, 2016 & Egypt & $30 / 59 / 60$ & $30 / 30 / 66.6$ & $30 / 53 / 50$ & - \\
\hline
\end{tabular}

HCC - hepatocellular carcinoma, LC - liver cirrhosis, $\mathrm{CH}$ - chronic hepatitis

one from Taiwan [9], one from China [13], and three from Egypt [14-16]. Altogether the studies included 373 HCC patients, 215 healthy controls, 132 cirrhotic patients and 92 patients with hepatitis. In a majority of studies, age and sex were matched between groups. One study [6] showed the mean of IL-10 on a graph from which we estimated it. Two studies $[12,14]$ reported SE and two studies $[11,13]$ reported median (range), which we changed to SD and mean $\pm S D$, respectively.

\section{Meta-analysis}

\section{HCC patients versus healthy controls}

Figure 2 shows a forest plot of the random effect of serum IL-10 level in the HCC patients compared with the healthy controls. The pooled analysis with continuous data concluded that IL-10 level in the patients was significantly higher than the controls $(\mathrm{MD}=6.96 ; 95 \%$ $\mathrm{CI}=4.91-9.01 ; p<0.00001)$ and $\mathrm{I}^{2}=89 \%(p<0.00001)$.

\section{HCC versus cirrhotic patients}

Figure 3 shows a forest plot of serum IL-10 level in the HCC patients compared with the cirrhotic patients. The pooled analysis showed that IL-10 level in the HCC patients was significantly higher than the cirrhotic patients $(\mathrm{MD}=2.92 ; 95 \% \mathrm{CI}=0.72-5.12 ; p=0.009)$ and $\mathrm{I}^{2}=89 \%(p<0.00001)$.

\section{HCC patients versus patients with hepatitis}

A forest plot of serum IL-10 level in the HCC patients compared with the patients with hepatitis is shown in Figure 4. The pooled analysis showed that the

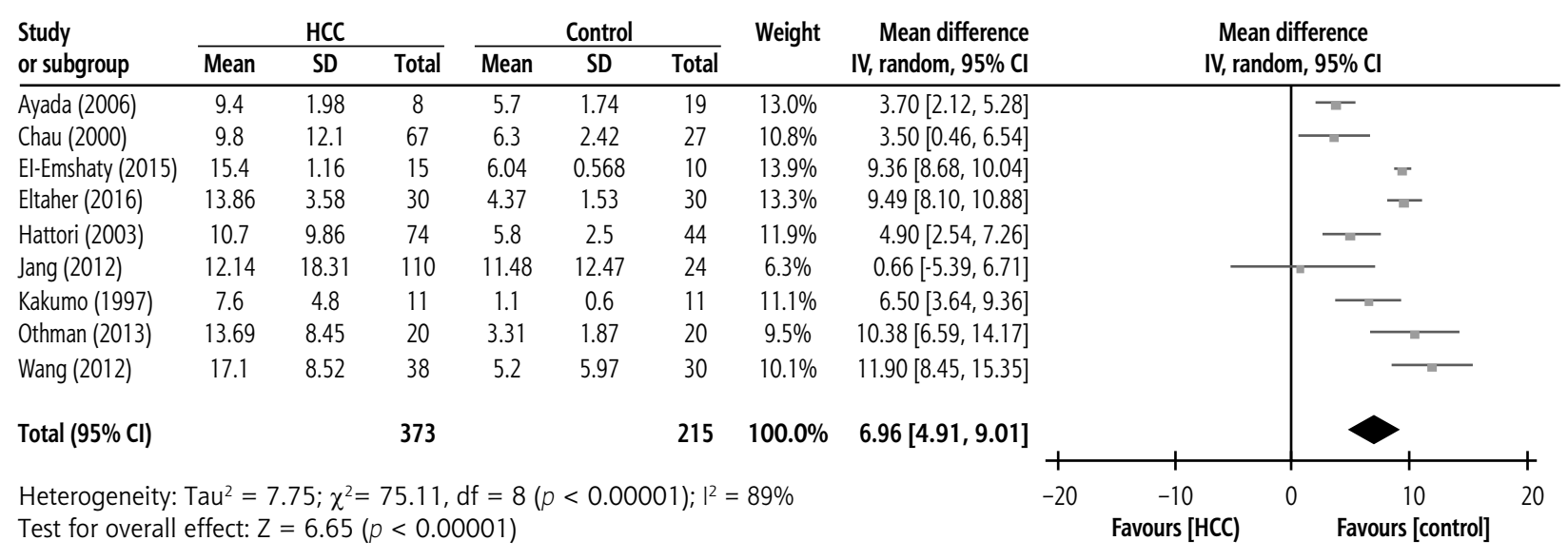

HCC - hepatocellular carcinoma

Fig. 2. Forest plot of random effect of serum IL-10 level in hepatocellular carcinoma patients compared with healthy controls $(n=9)$ 


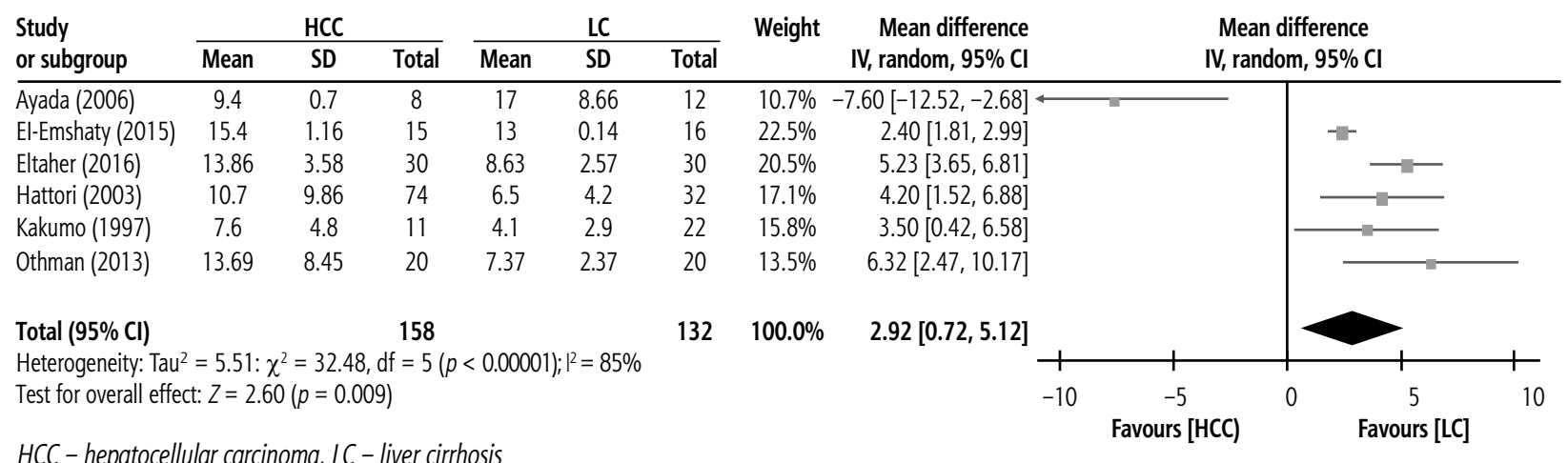

Fig. 3. Forest plot of random effect of serum IL-10 level in hepatocellular carcinoma patients compared with cirrhotic patients $(n=6)$

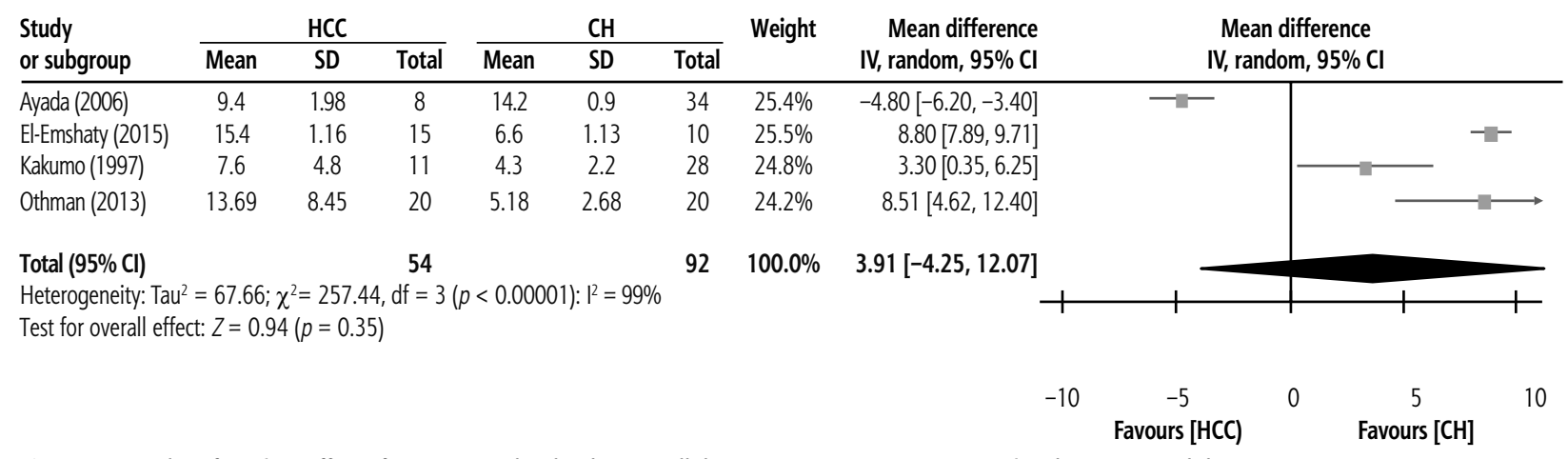

Fig. 4. Forest plot of random effect of serum IL-10 level in hepatocellular carcinoma patients compared with patients with hepatitis $(n=4)$
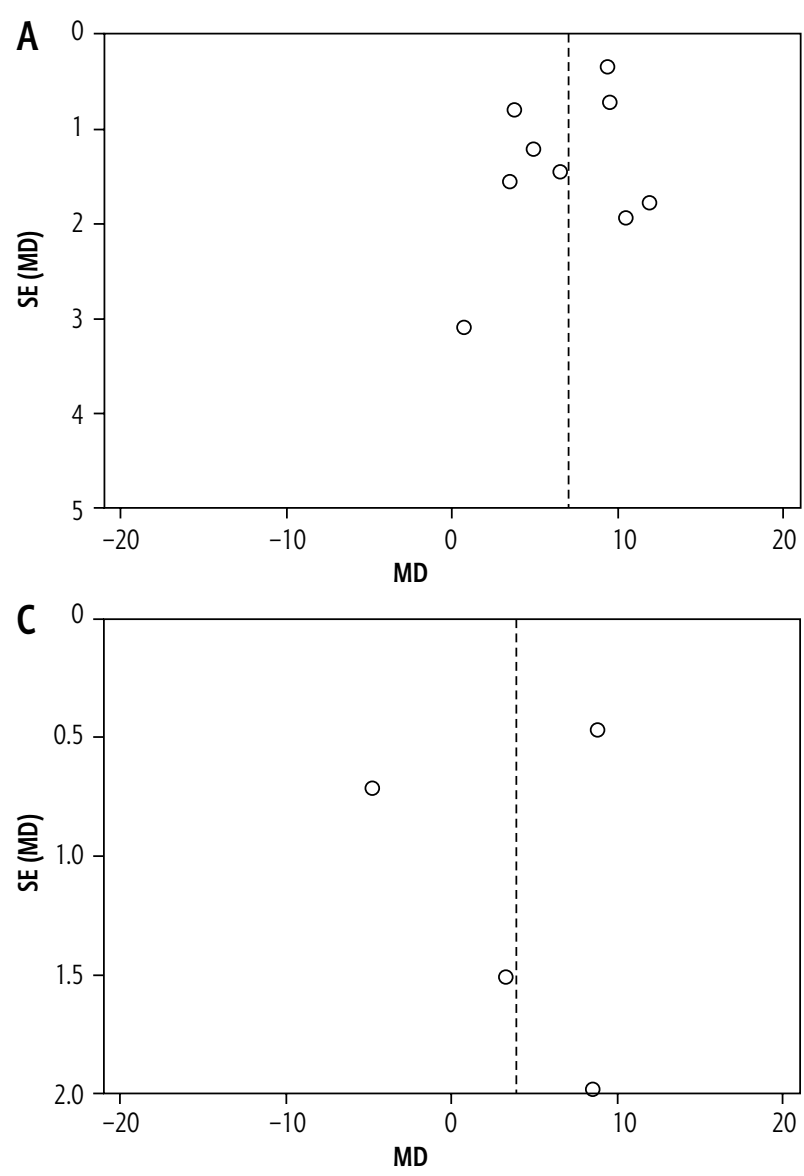

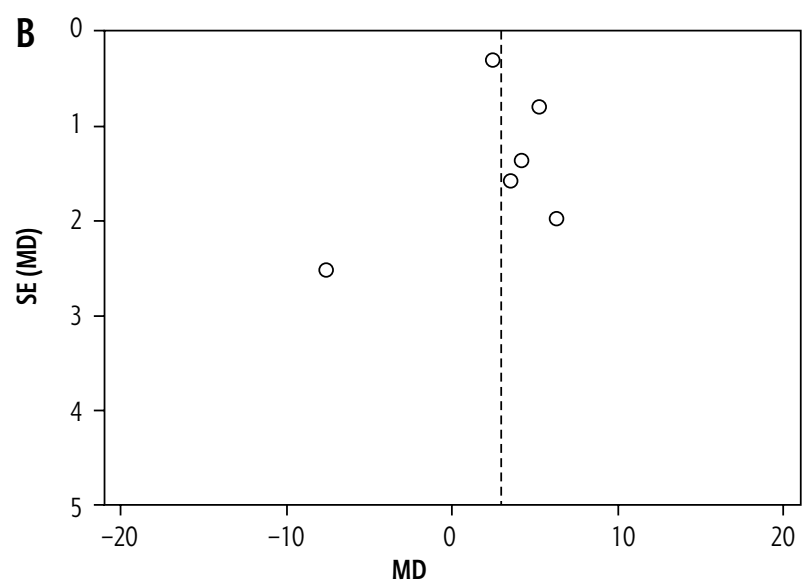

Fig. 5. Funnel plot of random effect of comparison of serum IL-10 levels in A) HCC patients and healthy controls, B) HCC and cirrhotic patients, C) HCC patients and patients with hepatitis 
level of this interleukin was similar in the two groups $(\mathrm{MD}=3.91 ; 95 \% \mathrm{CI}=-4.25-12.07 ; p=0.35)$ and $\mathrm{I}^{2}=99 \%$ $(p<0.00001)$.

\section{Publication bias}

A funnel plot for every analysis is shown in Figure 5. The results of the Begg's and Egger's tests revealed that no publication bias existed in terms of the comparison of serum IL-10 levels in the HCC and healthy control groups (Fig. 5A), the comparison of serum IL-10 levels in the HCC and cirrhosis groups (Fig. 5B), or the comparison of serum IL-10 levels in the HCC and hepatitis groups (Fig. 5C).

\section{Discussion}

The meta-analysis showed that serum IL-10 level in the HCC patients was significantly higher than in the healthy controls and cirrhotic patients, but not the patients with hepatitis. IL-10 is known as a pleiotropic cytokine with dual roles in the immune system, including immune suppression and immune stimulation [17]. Many studies have reported that serum IL-10 levels were higher in the HCC patients than in the healthy controls $[1,6,9,11-16,18]$ and that a higher IL-10 level was associated with worse prognosis [19]. Increased levels of IL-10 have been described as a negative prognostic indicator for survival in patients with various types of cancer $[9,18,20,21]$. These findings confirm a predominantly immunosuppressive role of IL-10 for circulating dendritic cells in patients with HCC, and thus may indicate novel aspects of tumor immune evasion [18]. IL-10 is an immune-suppressive cytokine and has been shown to inversely correlate with clinical outcome in patients with $\operatorname{HCC}[9,22]$. Alpha-fetoprotein (AFP) is the single most important tumor marker for HCC. Although the detection of serum AFP level is well established in screening and diagnostic purposes for HCC, a major shortcoming is that serum AFP is insensitive for early cancer detection [23]. IL-10 may help identify a subset of HCC patients with a low AFP level, and may serve as a complementary tumor marker in these patients [7]. Patients with a large $(>5 \mathrm{~cm})$ HCC more often had significantly higher (> $20 \mathrm{ng} / \mathrm{ml}$ ) AFP level and IL-10 expression [7].

Serum IL-10 levels have been observed to be significantly elevated in patients with type $\mathrm{C}$ chronic liver disease, and IL-10 may be related to the development of HCC [6]. Many studies have reported that serum IL-10 levels were higher in HCC patients than in patients with hepatitis $[6,14,15]$, but one study had opposite results [12]. IL-10 was higher in the serum and the supernatants of cultured peripheral blood monocyte cells (PBMCs) from HBV-infected patients [24]. Serum IL-10 levels were reportedly higher in those with HBV compared with controls [25, 26]. IL-10 plays key roles in the regulation of cellular immune response in HCV infection [27]. Some studies have found a significant association between higher IL-10 serum levels and HCV infection when compared to a healthy control group $[6,28,29]$.

The current findings suggested that an elevated IL-10 level may be related to hepatic injury caused by cirrhotic processes rather than tumor load. The authors concluded that the IL-10 level offers additional prognostic value to the existing tumor staging systems [30]. Many studies have reported that serum IL-10 levels were higher in HCC patients than in cirrhotic patients $[6,11$, 14-16], but one study had opposite results [12]. IL-10 was correlated with alanine transaminase (ALT) when analyzed in all the patients, but it was correlated with HBVDNA in chronic hepatitis and in liver cirrhosis [12].

\section{Limitations}

1. Combination of liver disease with HCC in some studies. 2. No matching of mean age between groups in several studies. 3. Low number of patients and controls in several studies. 4. High heterogeneity between the studies in each analysis, but there was no bias. 5. Difference in consumption of alcohol in the studies. 6. Difference in the Child-Pugh score and AFP levels for HCC patients and cirrhosis and hepatitis groups between the studies. 7. Hepatitis virus relation to cirrhotic patients and vice versa in some studies.

\section{Conclusions}

The increased IL-10 levels in the HCC patients compared with the cirrhotic patients and the healthy controls may show a significant role of this cytokine with elevated risk of HCC. In hepatitis the level is not significantly different from cancer, and thus it cannot be used as a tumor marker in hepatitis patients for evaluation of progression to HCC.

\section{Acknowledgments}

This article is part of a research project (code: 95549) supported by a grant from Kermanshah University of Medical Sciences Research Council.

\section{Disclosure}

Authors report no conflict of interest. 


\section{References}

1. Jang JW, Oh BS, Kwon JH, et al. Serum interleukin-6 and C-reactive protein as a prognostic indicator in hepatocellular carcinoma. Cytokine 2012; 60: 686-693.

2. Xue H, Lin F, Tan H, et al. Overrepresentation of IL-10-Expressing B Cells Suppresses Cytotoxic CD4+ T Cell Activity in HBV-Induced Hepatocellular Carcinoma. PLoS One 2016; 11: e0154815.

3. Di Bisceglie AM. Hepatitis c and hepatocellular carcinoma. Hepatology 1997; 26: 34S-85S.

4. Wan L, Kung YJ, Lin YJ, et al. Th1 and Th2 cytokines are elevated in HCV-infected SVR(-) patients treated with interferon- $\alpha$. Biochem Bioph Res Co 2009; 379: 855-860.

5. Naugler WE, Karin M. The wolf in sheep's clothing: the role of interleukin-6 in immunity. Inflammation and cancer. Trends Mol Med 2008; 14: 109-119.

6. Kakumu S, Okumura A, Ishikawa T, et al. Serum levels of IL-10, IL-15 and soluble tumor necrosis factor-alpha (TNF-a) receptors in type C chronic liver disease. Clin Exp Immunol 1997; 109: 458-463.

7. Hsia CY, Huo TI, Chiang SY, et al. Evaluation of interleukin-6, interleukin-10 and human hepatocytegrowth factor as tumor markers for hepatocellular carcinoma. Eur J Surg Oncol 2007; 33: 208-212.

8. Fortis C, Foppoli M, Gianotti L, et al. Increased interleukin-10 serum levels in patients with solid tumours. Cancer Lett 1996; 104: 1-5.

9. Chau GY, Wu CW, Lui WY, et al. Serum interleukin-10 but not interleukin-6 is related to clinical outcome in patients with resectable hepatocellular carcinoma. Ann Surg 2000; 231: 552-558.

10. Hozo SP, Djulbegovic B, Hozo I. Estimating the mean and variance from the median, range, and the size of a sample. BMC Med Res Methodol 2005; 5: 13.

11. Hattori E, Okumoto K, Adachi T, et al. Possible contribution of circulating interleukin-10 (IL-10) to anti-tumor immunity and prognosis in patients with unresectable hepatocellular carcinoma. Hepatol Res 2003; 27: 309-314.

12. Ayada M, Ishikawa T, Okumura A, et al. Alteration of serum cytokine balances among different phases of chronic hepatitis $\mathrm{B}$ virus infection. Hepatol Res 2006; 34: 214-221.

13. Wang XD, Wang L, Ji FJ, et al. Decreased CD27 on B Lymphocytes in Patients with Primary Hepatocellular Carcinoma. Decreased $\mathrm{CD} 27$ on B lymphocytes in patients with primary hepatocellular carcinoma. J Int Med Res 2012; 40: 307-316.

14. Othman MS, Aref AM, Mohamed AA, et al. Serum Levels of Interleukin- 6 and Interleukin-10 as Biomarkers for Hepatocellular Carcinoma in Egyptian Patients. ISRN Hepatol 2013; 2013: 412317.

15. El-Emshaty HM, Nasif WA, Mohamed IE. Serum Cytokine of IL-10 and IL-12 in Chronic Liver Disease: The Immune and Inflammatory Response. Dis Markers 2015; 2015: 707254.

16. Eltaher SM, El-Gil R, Fouad N, et al. Evaluation of serum levels and significance of soluble CD40 ligand in screening patients with hepatitis $C$ virus-related hepatocellular carcinoma. East Mediterr Health J 2016; 22: 603-610.

17. Mocellin S, Panelli MC, Wang E, et al. The dual role of IL-10. Trends Immunol 2003; 24: 36-43.

18. Beckebaum S, Zhang X, Chen X, et al. Increased levels of interleukin-10 in serum from patients with hepatocellular carcinoma correlate with profound numerical deficiencies and immature phenotype of circulating dendritic cell subsets. Clin Cancer Res 2004; 10: 7260-7209.
19. Zhao S, Wu D, Wu P, et al. Serum IL-10 Predicts Worse Outcome in Cancer Patients: A Meta-Analysis. PLoS One 2015; 10: e0139598.

20. Shin HD, Park BL, Kim LH, et al. Interleukin 10 haplotype associated with increased risk of hepatocellular carcinoma. Hum Mol Genet 2003; 12: 901-906.

21. Salgado R, Junius S, Benoy I, et al. Circulating interleukin-6 predicts survival in patients with metastatic breast cancer. Int J Cancer 2003; 103: 642-646.

22. Achyut BR, Yang L. Transforming growth factorbeta in the gastrointestinal and hepatic tumor microenvironment. Gastroenterology 2011; 141: 1167-1178.

23. Zakhary NI, El-Merzabani MM, El-Sawi NM, et al. Impact of different biochemical markers in serum of patients with benign and malignant liver diseases. J Adv Res 2011; 2: 49-55.

24. Ji W, Wang HF, Feng CQ. Activation-induced cell death in peripheral blood mononuclear cells (PBMCs) from patients with chronic hepatitis B may be related to abnormal production of interleukin 12 and 10. J Viral Hepat 2001; 8: 30-33.

25. Tulek N, Saglam SK, Saglam M, et al. Soluble interleukin-2 receptor and interleukin-10 levels in patients with chronic hepatitis B infection. Hepatogastroenterology 2000; 47: 828-831.

26. Wang FS, Xing LH, Liu MX, et al. Dysfunction of peripheral blood dendritic cells from patients with chronic hepatitis B virus infection. World J Gastroenterol 2001; 7: 537-544.

27. Aroucha DC, do Carmo RF, Moura P, et al. High tumor necrosis factor- $\alpha$ /interleukin-10 ratio is associated with hepatocellular carcinoma in patients with chronic hepatitis C. Cytokine 2013; 62: 421-425.

28. Akcam FZ, Tigli A, Kaya O, et al. Cytokine levels and histopathology in chronic hepatitis B and chronic hepatitis C. J Interferon Cytokine Res 2012; 32: 570-574.

29. Abayli B, Canatarog `lu A, Akkiz H. Serum profile of T helper 1 and $\mathrm{T}$ helper 2 cytokines in patients with chronic hepatitis $\mathrm{C}$ virus infection. Turk J Gastroenterol 2003; 14: 7-11.

30. Chan SL, Mo FK, Wong CS, et al. A study of circulating interleukin 10 in prognostication of unresectable hepatocellular carcinoma. Cancer 2012; 118: 3984-3992. 